\title{
Relation between quantitative coronary CTA and myocardial ischemia by adenosine stress myocardial CT perfusion
}

\author{
Alexander R. van Rosendael, MD, ${ }^{a, b}$ Lucia J. Kroft, $M D, P h D,{ }^{c}$ \\ Alexander Broersen, PhD, ${ }^{\mathrm{d}}$ Jouke Dijkstra, PhD, ${ }^{\mathrm{d}}$ Inge J. van den Hoogen, MSc, ${ }^{\mathrm{a}}$

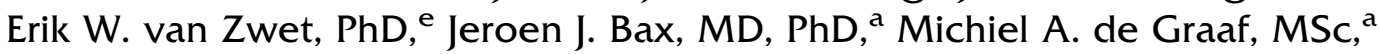 \\ and Arthur J. Scholte, MD, PhD ${ }^{\mathrm{a}}$ \\ a Department of Cardiology, Heart and Lung Center, Leiden University Medical Center, Leiden, \\ The Netherlands \\ ${ }^{\mathrm{b}}$ The Interuniversity Cardiology Institute of the Netherlands, Utrecht, The Netherlands \\ c Department of Radiology, Leiden University Medical Center, Leiden, The Netherlands \\ d Division of Image Processing, Department of Radiology, Leiden University Medical Center, \\ Leiden, The Netherlands \\ e Department of Medical Statistics and Bio-informatics, Leiden University Medical Center, \\ Leiden, The Netherlands
}

Received Nov 19, 2015; accepted Dec 22, 2015

doi: $10.1007 / \mathrm{s} 12350-016-0393-7$

Background. Coronary-computed tomography angiography (CTA) has limited accuracy to predict myocardial ischemia. Besides luminal area stenosis, other coronary plaque morphology and composition parameters may help to assess ischemia. With the integration of coronary CTA and adenosine stress CT myocardial perfusion (CTP), reliable information regarding coronary anatomy and function can be derived in one procedure. This analysis aimed to investigate the association between coronary stenosis severity, plaque composition and morphology and the presence of ischemia measured with adenosine stress myocardial CTP.

Methods and Results. 84 patients (age, $62 \pm 10$ years; $48 \%$ men) who underwent sequential coronary CTA and adenosine stress myocardial CT perfusion were analyzed. Automated quantification was performed in all coronary lesions (quantitative CTA). Downstream myocardial ischemia was assessed by visual analysis of the rest and stress CTP images and defined as a summed difference score of $\geq 1$. One or more coronary plaques were present in 146 coronary arteries of which $31(21 \%)$ were ischemia-related. Of the lesions with a stenosis percentage $<50 \%, 50 \%-70 \%$, and $>70 \%$, respectively, $9 \%(6 / 67), 18 \%(9 / 51)$, and $57 \%(16 / 28)$ demonstrated downstream ischemia. Furthermore, mean plaque burden, plaque volume, lesion length, maximal plaque thickness, and dense calcium volume were significantly higher in ischemia-related lesions, but only stenosis severity $(\%)(\mathrm{OR} 1.06 ; 95 \%$ CI 1.02-1.10; $P=.006)$ and lesion length $(\mathrm{mm})(\mathrm{OR} 1.26 ; 95 \%$ CI 1.02-1.55; $P=.029)$ were independent correlates.

Conclusions. Increasing stenosis percentage by quantitative CTA is positively correlated to myocardial ischemia measured with adenosine stress myocardial CTP. However, stenosis percentage remains a moderate determinant. Lumen area stenosis and lesion length were independently associated with ischemia, adjusted for coronary plaque volume, mean plaque burden, maximal lesion thickness, and dense calcium volume. (J Nucl Cardiol 2017;24:1253-62.)

Reprint requests: Arthur J. Scholte, MD, PhD, Department of Cardiology, Heart and Lung Center, Leiden University Medical Center, Albinusdreef 2, 2333 ZA, 2300 RC Leiden, The Netherlands; a.j.h.a.scholte@lumc.nl
$1071-3581 / \$ 34.00$

Copyright $₫ 2016$ The Author(s). This article is published with open access at Springerlink.com 
Key Words: Coronary artery disease $\cdot$ quantitative coronary CTA $\cdot$ myocardial CT perfusion $\cdot$ imaging $\cdot$ myocardial ischemia

\begin{tabular}{|c|c|}
\hline \multicolumn{2}{|c|}{ Abbreviations } \\
\hline APV & Aggregate plaque volume \\
\hline CTA & Computed tomography angiography \\
\hline СТP & $\begin{array}{l}\text { Myocardial-computed tomography } \\
\text { perfusion }\end{array}$ \\
\hline FFR & Fractional flow reserve \\
\hline ICA & Invasive coronary angiography \\
\hline LAD & $\begin{array}{l}\text { Left anterior descending coronary } \\
\text { artery }\end{array}$ \\
\hline LCX & Left circumflex coronary artery \\
\hline MLA & Minimal lumen area \\
\hline RCA & Right coronary artery \\
\hline SPECT & $\begin{array}{l}\text { Single-photon emission-computed } \\
\text { tomography }\end{array}$ \\
\hline
\end{tabular}

See related editorial, pp. 1263-1266

\section{INTRODUCTION}

Coronary-computed tomography angiography (CTA) is an established technique to detect or rule out coronary artery disease (CAD) and correlates well with invasive coronary angiography (ICA). ${ }^{1,2}$ However, obstructive $\mathrm{CAD}$ ( $\geq 50 \%$ luminal narrowing) detected by coronary CTA has limited value to predict myocardial ischemia. ${ }^{3}$ Moreover, hemodynamically significant stenosis in patients with stable CAD is important for prognosis and the need for revascularization. ${ }^{4}$ Previous studies demonstrated that besides luminal area stenosis, additional plaque characteristics are related to myocardial ischemia. ${ }^{5,6}$ Quantification of stenosis severity, plaque composition and morphology can nowadays be performed with dedicated post-processing data software (quantitative CTA). ${ }^{7}$ Until recently, the hemodynamic consequence of an obstructive lesion detected by coronary CTA was obtained by additional nuclear or echocardiographic ischemia testing or invasive fractional flow reserve (FFR) measurement. A relatively new technique, which can be performed in addition to coronary CTA in the same setting, is adenosine stress myocardial CT perfusion (CTP) which provides functional information of a coronary stenosis. ${ }^{8}$

The relationship between luminal narrowing, additional coronary plaque characteristics measured with quantitative CTA, and myocardial ischemia detected by adenosine stress myocardial CTP is unknown. Accordingly, the present study aimed to explore the association of quantitatively assessed coronary plaque characteristics with myocardial ischemia by adenosine stress CTP.

\section{METHODS}

\section{Patients}

The population consisted of 115 consecutive patients with new onset chest pain who were referred from the outpatient clinic from March 2013 till December 2014 for cardiac CT. All patients underwent coronary CTA and subsequently adenosine stress CTP at the same day. Until March 2014, all referred patients underwent both scans. After March 2014, directly after the acquisition of the coronary CTA, the presence of obstructive CAD ( $\geq 50 \%$ stenosis) was assessed on-site by an experienced physician. Only in case of obstructive CAD, additional adenosine stress CTP was performed. Patients with previous myocardial infarction, percutaneous coronary intervention, coronary artery bypass graft, anomalous coronary arteries, or insufficient image quality in one or both CT scans were excluded. Contraindications were atrial fibrillation, renal insufficiency, second or third degree atrioventricular block, known allergy to iodine-containing contrast agents and pregnancy. Clinical data were prospectively entered into the departmental electronic information system (EPDVision $\odot$, Leiden University Medical Center, The Netherlands) and retrospectively analyzed. According to the Dutch law, no Institutional Review Board approval is required for this retrospective analysis of clinically acquired data.

\section{Cardiac CT Acquisition}

Sequential coronary CTA and adenosine stress myocardial CTP were both performed using a 320 detector row volumetric scanner (Aquilion ONE, Toshiba Medical Systems, Otawara, Japan). The effective radiation exposure was calculated by multiplying the dose-length-product by $0.014 \mathrm{mSv} \cdot \mathrm{mGy}^{-1} \cdot \mathrm{cm}^{-1}$.

\section{Coronary CTA Acquisition}

Patients with a heart rate exceeding 60 beats $\cdot$ minute $^{-1}$ received $25-150 \mathrm{mg}$ of oral metoprolol 1 hour before the scan, unless contraindicated. If the heart rate remained above 60 beats.minute ${ }^{-1}$, up to $15 \mathrm{mg}$ of intravenous metoprolol was administrated additionally. First, a low-dose non-contrast enhanced scan was performed to determine the coronary artery calcium score and to assess the needed coverage (120$160 \mathrm{~mm}$ ) depending on the craniocaudal length of the heart. The coronary CTA was performed with a peak tube voltage between 100 and $135 \mathrm{kV}$ and tube current between 140 and $580 \mathrm{~mA}$, depending on body habitus. The detector collimation was $320 \times 0.5 \mathrm{~mm}$, gantry rotation time was $350 \mathrm{~ms}$, and temporal resolution was $175 \mathrm{~ms}$. The contrast agent (Iomeron 400, Bracco, Milan, Italy) was injected in a triphasic contrast 
injection protocol: first, $50-90 \mathrm{~mL}$ (depending on patient weight) contrast agent (flow rate $5-6 \mathrm{~mL} \cdot \mathrm{s}^{-1}$ ), followed by $20 \mathrm{~mL}$ of a 1:1 mixture of contrast and saline and finally $25 \mathrm{~mL}$ of saline (flow rate $3 \mathrm{~mL} \cdot \mathrm{s}^{-1}$ ). Amounts and injection protocol were equal for coronary CTA and adenosine stress CTP and varied between 60 and $90 \mathrm{~mL}$ based on patient weight. Prospective ECG triggering was used to cover $70 \%$ $80 \%$ of the R-R interval. In patients with a heart rate $>65$ beats.minute ${ }^{-1}$ or irregular heart rate, $30 \%-80 \%$ of the R-R interval was covered. Real-time bolus tracking was performed in the descending aorta, with a threshold of 300 Hounsfield Units (HU). Reconstructed left ventricle data acquired from the coronary CTA scan served as the rest myocardial perfusion study.

\section{Adenosine Stress Myocardial CTP Acquisition}

At least 20 minutes after finishing the coronary CTA scan, adenosine $\left(0.14 \mathrm{mg} \cdot \mathrm{kg}^{-1} \cdot\right.$ minute $\left.^{-1}\right)$ was administered intravenously during continuous ECG monitoring. After 4 minutes of adenosine infusion, contrast agent was administrated. Once the target threshold of $300 \mathrm{HU}$ was reached in the descending aorta, adenosine stress myocardial CTP images were acquired the next one to two heartbeats depending on patient heart rate, covering $80 \%-99 \%$ of the $\mathrm{R}-\mathrm{R}$ interval. The tube settings were the same as for the coronary CTA.

\section{Quantitative CTA}

Quantitative CTA was performed to provide a detailed and objective assessment of the coronary plaque. Dedicated software (QAngio CT Research Edition; Medis Medical Imaging Systems, Leiden, The Netherlands) was used to perform the automated quantitative analysis. As previously described, the software automatically identifies the vessels and detects the contours of the vessel wall and the lumen. ${ }^{5,7}$ If needed, the observer could manually adjust these contours. Automated quantification for each coronary lesion in all coronary arteries was performed. Reference lines for the lumen and the outer vessel wall were created using non-bifurcated, non-diseased segments proximal and distal to the lesion. Subsequently, a reference slope for lumen and outer vessel wall was created between these reference segments, which served as a reference frame of a non-diseased artery. This reference slope represented the normal proximal to distal tapering of a coronary artery. Quantitative CTA parameters were automatically generated by using the plaque contours in relation to the reference slope. In the absence of coronary plaques, no analysis was performed and these arteries were excluded from further analysis. Percentage lumen area stenosis, lumen diameter stenosis, mean plaque burden, plaque volume, lesion length, maximal plaque thickness, fibrous volume, fibro-fatty volume, necrotic core volume, dense calcium volume, and remodeling index were derived from each coronary plaque. Plaque constitution (fibrous, fibrofatty, necrotic core, and dense calcium) was automatically assessed using adaptive HU thresholds: meaning that HU thresholds for plaque composition are adapted according to lumen contrast attenuation variations, as demonstrated previously. ${ }^{10}$ Definitions of the parameters are presented in Table 1 .

The most severe lesion in each coronary artery was selected by maximum lumen area stenosis percentage. Coronary arteries were defined as left anterior descending artery (LAD), right coronary artery (RCA), and left circumflex artery (LCX). Plaques in diagonal branches were allocated to the $\mathrm{LAD}$, and plaques in the intermediate branch were allocated to the LCX. Plaque characteristics were compared between ischemia-related and non-ischemic coronary lesions. To clarify a possible incremental relation with myocardial ischemia, lumen area stenosis was divided into three groups $(<50 \%$, $50 \%-70 \%$, and $>70 \%)$. $^{11}$

\section{Adenosine Stress and Rest Myocardial CTP Analysis}

All myocardial CTP images were analyzed and interpreted by two trained observers with dedicated post-processing software (Vitrea FX 6.5; Vital Images, Minnetonka, Minnesota, USA). Coronary CTA and myocardial perfusion analyses were done independently from each other to reduce bias caused by knowledge of the other scan result. Data were arranged in the short axis, vertical long axis, and horizontal long axis with a slice thickness of $3 \mathrm{~mm}$. Data from the coronary CTA were used for the rest myocardial CTP. The phase with the best image quality was selected and interpreted with a narrow window width and level setting (W300/L150), according to the 17-myocardial segment model. ${ }^{12}$ The best phase was defined as the phase with the least artifacts. The observers were allowed to adjust the display settings after the initial exploratory reading. Each segment was scored for the presence of perfusion defects. Inter-observer variability was dissolved by consensus.

Fixed defects were defined as the persistence of a perfusion defect in the same myocardial segment at rest and during adenosine stress myocardial CTP. In case of an abnormal scan, all phases (both systolic and diastolic) were examined to better differentiate between potential artifacts and real perfusion defects. The persistence of a hypo-enhanced area in multiple phases suggests true perfusion defect, as a motion artifact usually does not persist in multiple phases. ${ }^{13}$ To assess the hemodynamic significance of each coronary lesion, corresponding myocardial ischemia was defined as a summed difference score by adenosine stress CTP $\geq 1$. Each myocardial segment was matched to its corresponding epicardial coronary artery, using a standard model. ${ }^{12}$ The anteroseptal and anterior segments were matched to the $\mathrm{LAD}$, the lateral to the LCX, and the inferoseptal and inferior segments to the RCA. If a mismatch existed while using the standard coronary artery-myocardial segment alignment and multiple arteries were supplying the ischemic myocardium based on vessel tracking, the artery with obstructive CAD was related to the perfusion defect. Three-dimensional fusion of coronary CTA and adenosine stress myocardial CTP images by 
Table 1. Definitions of quantitative CTA-derived parameters

\begin{tabular}{|c|c|}
\hline Parameter & Definition \\
\hline Lumen area stenosis (\%) & 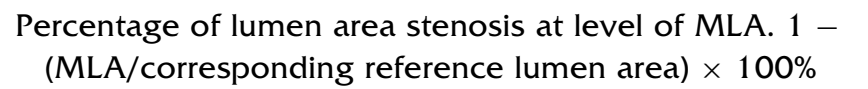 \\
\hline Lumen diameter stenosis (\%) & Percentage diameter stenosis at the MLA \\
\hline Mean plaque burden (\%) & $\begin{array}{l}\text { Sum of ((vessel wall area - lumen area)/vessel wall area) } \\
\text { per slice/number of slices }\end{array}$ \\
\hline Lesion length $(\mathrm{mm})$ & Distance between proximal and distal ends of the plaque \\
\hline Maximal plaque thickness (mm) & Maximal distance between vessel wall and lumen \\
\hline Remodeling index & $\begin{array}{l}\text { Vessel wall area/corresponding reference vessel wall area } \\
\text { at the level of the MLA }\end{array}$ \\
\hline
\end{tabular}

CTA, computed tomography angiography; MLA, minimal lumen area

Vitrea software was used to facilitate the linking of regional myocardial ischemia with its corresponding coronary artery, as depicted in Figure 1.

\section{Statistical Analysis}

Continuous data were presented as mean \pm standard deviation. When non-normally distributed, data were presented as medians with 25 th and 75 th percentiles. Categorical data were presented as frequencies and percentages and were compared with the Chi square test. Quantitative CTA parameters of lesions with and without downstream myocardial ischemia were analyzed with the independent-samples $t$ test or the Mann-Whitney test, as appropriate. Multivariate logistic regression analysis was performed with the plaque characteristics that were significantly different between ischemic and non-ischemic lesions; ischemia was the dependent variable. Values were expressed as odds ratios (OR) with $95 \%$ confidence interval (CI). A two-sided $P$ value less than .05 was considered statistically significant. All statistical analyses were performed with the use of IBM SPSS Statistics software (version 20, IBM Corp, Armonk, New York, USA).

\section{RESULTS}

\section{Patients}

A total of 115 patients underwent both coronary CTA and adenosine stress myocardial CTP. Excluded were 31 patients, because of insufficient image quality $(\mathrm{n}=12)$, previous PCI $(\mathrm{n}=8)$ or CABG $(\mathrm{n}=3)$, anomalous coronary arteries $(n=6)$, and the presence of a fixed perfusion defect (indicating prior myocardial infarction) by myocardial CTP $(n=2)$. The 84 remaining patients were included in the present analysis. Clinical characteristics are presented in Table 2 . In total, $40(48 \%)$ patients were men and the mean age was $62( \pm 10)$ years. The prevalence of risk factors for coronary artery disease was high: $32 \%$ had diabetes, 58\% hypertension, $44 \%$ hypercholesterolemia, $13 \%$ were currently smoking, and $50 \%$ had a family history of CAD. The median calcium score was 98 (IQR 19-330). Effective radiation exposure for the coronary CTA was $2.6 \mathrm{mSv}$ (IQR: 1.7-3.7) and for adenosine stress CTP: $3.2 \mathrm{mSv}$ (IQR: 2.3-4.6). The dose for both scans was $6.4 \mathrm{mSv}$ (IQR: 4.3-8.6).

\section{Quantitative CTA}

Of the 252 coronary arteries in total, at least one coronary plaque (calcified, mixed or non-calcified) was present in $146(58 \%)$ arteries. 67 (42\%) plaques showed a stenosis $<50 \%$; 51 (35\%) plaques between 50 and $70 \%$ and $28(19 \%)$ plaques $>70 \%$ stenosis. 106 arteries did not show any coronary lesion. Downstream those, 2 perfusion defects were seen.

\section{Adenosine Stress Myocardial CTP}

In total, $31(21 \%)$ of the 146 coronary lesions were related to corresponding myocardial ischemia by adenosine stress CTP. The remaining 115 (79\%) lesions did not demonstrate downstream ischemia. Compared with non-ischemic lesions, hemodynamic significant lesions showed a significantly higher percentage lumen area stenosis $(69.0 \% \pm 16.8$ vs $49.6 \% \pm 17.2, P<.001)$, lumen diameter stenosis $(46.7 \% \pm 16.8$ vs $30.2 \% \pm$ $13.1, P<.001)$, mean plaque burden $(59.6 \% \pm 8.7$ vs $52.3 \% \pm 9.9, \quad P<.001)$, plaque volume $\left(68.7 \mathrm{~mm}^{3}\right.$ [40.2-126.0] vs $44.7 \mathrm{~mm}^{3}$ [24.1-80.3], $\left.P=.021\right)$, lesion length $\quad(12.3 \mathrm{~mm} \quad$ [4.9-14.9], vs 6.5 [4.0-10.4], $P=.033)$, maximal plaque thickness $(2.3 \mathrm{~mm}$ [1.82.7] vs $1.9[1.5-2.4], P=.021)$, and dense calcium volume (25.8 [7.6-39.4] vs $7.8[0.0-20.2], P=.005)$, as presented in Table 3.

In multivariate analysis, lumen area stenosis (\%) (OR 1.06; 95\% CI 1.02-1.10; $P=.006$ ) and lesion length $(\mathrm{mm})(\mathrm{OR} 1.26 ; 95 \%$ CI $1.02-1.55 ; P=.029)$ 
A

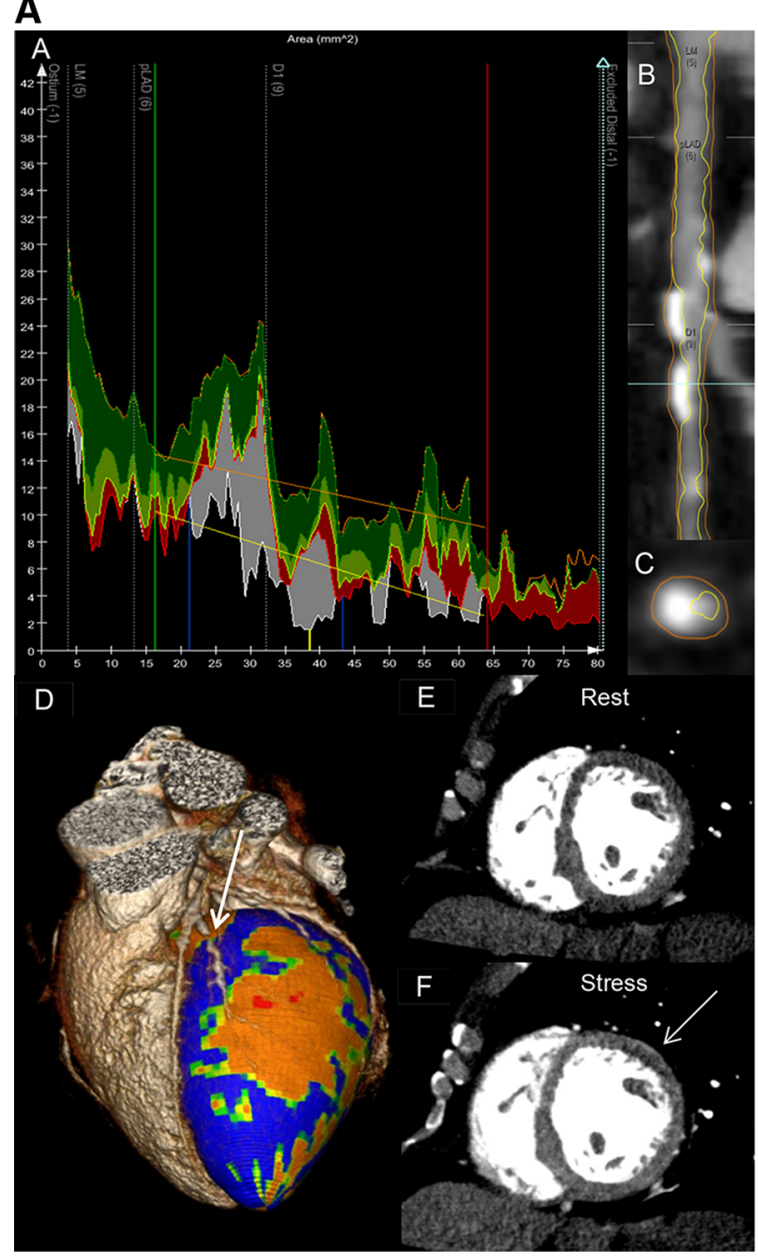

B

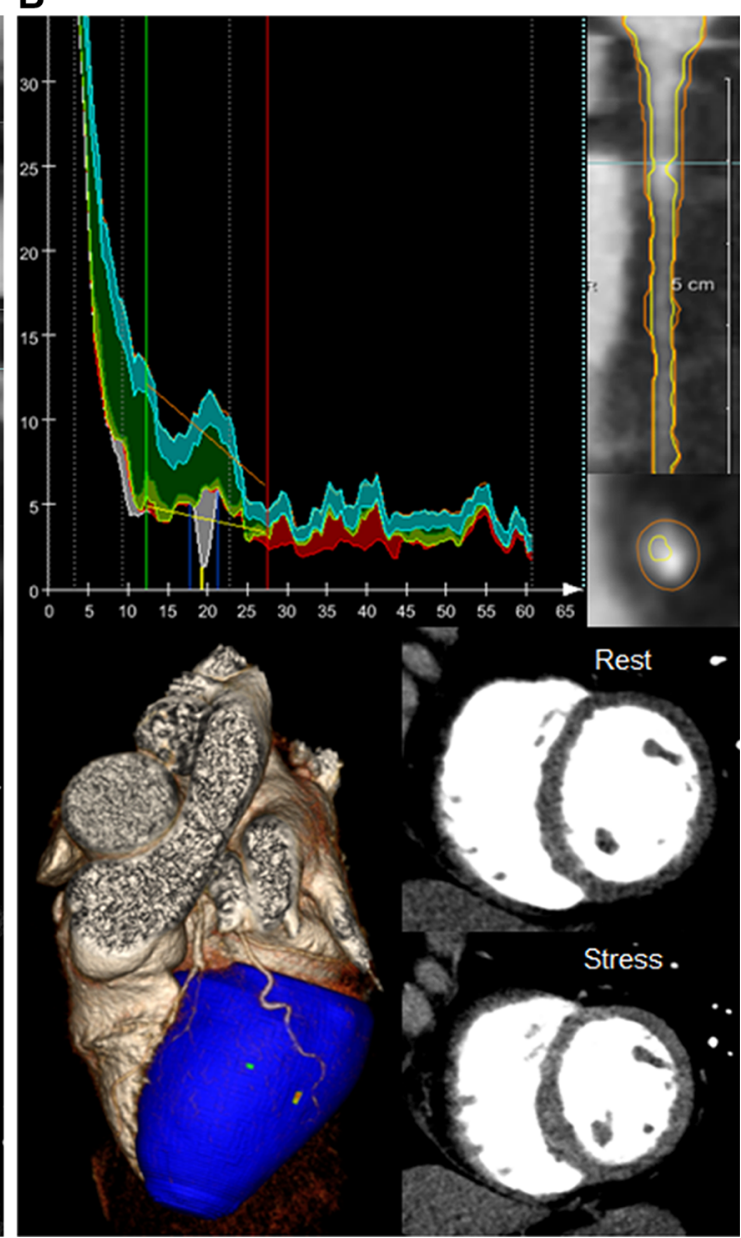

Figure 1. (A) Quantitative CTA and adenosine stress CTP of an ischemic lesion. Example of a 58year-old female patient with a lesion in the first diagonal branch and corresponding myocardial ischemia. A Automated quantitative CTA of the artery was performed. The blue lines were set proximal and distal to the lesion. The green and red lines represent non-diseased coronary artery segments proximal and distal to the lesion. The yellow and orange lines represent the reference markers for, respectively, the lumen and vessel wall. The vertical yellow line is placed at the site of maximal stenosis percentage: $76.6 \%$. Furthermore, mean plaque burden was $79.9 \%$; plaque volume: $129.3 \mathrm{~mm}^{3}$; lesion length: $19.0 \mathrm{~mm}$; maximal plaque thickness: $2.6 \mathrm{~mm}$; dense calcium volume: $36.6 \mathrm{~mm}^{3} . B$ Longitudinal lumen and vessel wall contours. $C$ Transverse lumen and vessel wall contours at the site of maximal stenosis percentage. $D$ 3D fusion of the coronary CTA and myocardial hypo-perfusion during adenosine stress (orange, red). A stenosis in the first diagonal branch (arrow) is depicted with corresponding myocardial ischemia. E Rest CTP study showing normal myocardial enhancement. $F$ Adenosine stress myocardial CTP showing a small anterolateral subendocardial perfusion defect (arrow). (B) Quantitative CTA and adenosine stress CTP of a nonischemic lesion. Same data reconstructions as shown in (A). A non-ischemic coronary lesion in the proximal LCX is depicted. Maximal stenosis percentage was: $69.1 \%$. Mean plaque burden was: 67.2\% ; plaque volume: $27.1 \mathrm{~mm}^{3}$; lesion length: $3.5 \mathrm{~mm}$; maximal plaque thickness: $1.8 \mathrm{~mm}$; dense calcium volume: $9.4 \mathrm{~mm}^{3}$. Despite the high stenosis percentage, lesion length, maximal plaque thickness, and dense calcium volume were relatively low, resulting in normal myocardial enhancement on adenosine stress.

were independently correlated to ischemia, when entered in the model with maximal plaque thickness (mm) (OR 1.61; 95\% CI 0.50-5.18; $P=.427)$, dense calcium volume $\left(\mathrm{mm}^{3}\right)$ (OR $0.99 ; 95 \%$ CI $0.95-1.04$;
$P=.804)$, mean plaque burden $(\%)(\mathrm{OR} 1.04 ; 95 \% \mathrm{CI}$ $0.96-1.13 ; P=.325)$, and plaque volume $\left(\mathrm{mm}^{3}\right)$ (OR $0.98 ; 95 \%$ CI $0.96-1.00 ; P=.089)$. An example of a patient with a severely stenotic lesion plus high values 
Table 2. Clinical characteristics

\begin{tabular}{ll}
\hline \multicolumn{1}{c}{$\begin{array}{c}\text { Baseline characteristics } \\
(\mathbf{n}=\mathbf{8 4 )}\end{array}$} & Values \\
\hline Men & $40(48)$ \\
Age (years) & $62 \pm 10$ \\
Calcium score & $98(19-330)$ \\
Diabetes & $27(32)$ \\
Hypertension* & $49(58)$ \\
Hypercholesterolemia ${ }^{\dagger}$ & $37(44)$ \\
Current smoking & $11(13)$ \\
Positive family history ${ }^{\star}$ & $42(50)$ \\
Beta-blocker & $40(48)$ \\
ACE-I/ARB & $39(46)$ \\
Calcium antagonist & $16(19)$ \\
Statin & $38(45)$ \\
Acetyl salicylic acid & $25(30)$
\end{tabular}

Data are represented as mean \pm standard deviation, median (IQR), or as number (percentage).

$A C E-I$, Angiotensin converting enzyme inhibitor; $A R B$, angiotensin receptor blocker.

* Systolic blood pressure $\geq 140 \mathrm{~mm} \mathrm{Hg}$, diastolic blood pressure $\geq 90 \mathrm{~mm} \mathrm{Hg}$, or use of antihypertensive mediation.

Serum total cholesterol $\geq 230 \mathrm{mg} \cdot \mathrm{dL}^{-1}$ or serum triglycerides $\geq 200 \mathrm{mg} \cdot \mathrm{dL}^{-1}$ or treatment with lipid-lowering drugs.

$\$$ Presence of CAD in 1 st degree family members at $<55$ years in men and $<65$ years in women.

of mean plaque burden, plaque volume, lesion length, maximal plaque thickness, and dense calcium volume that were related to ischemia is shown in Figure1A. Figure 1B demonstrates a lesion with high stenosis percentage but relatively low plaque volume, maximal plaque thickness, lesion length, and dense calcium volume that did not cause ischemia.

\section{Association Between Stenosis Severity and Adenosine Stress Myocardial CTP}

$9 \%(6 / 67)$ of the coronary lesions $<50 \%$ stenosis were related to myocardial ischemia. In the categories, $50 \%-70 \%$ and $>70 \%$, respectively, $18 \%(9 / 51)$ and $57 \%$ $(16 / 28)$ were ischemic, as depicted in Figure 2A. Of 12 of the non-ischemic lesion $>70 \%$ stenosis, only 2 were located in proximal coronary parts and 6 were located distally in side branches (intermediate, anterolateral, obtuse marginal, or diagonal branches) which may have had influence on the stenosis-ischemia relationship. In a per vessel analysis, a worse correlation between stenosis severity and ischemia was seen in the lesions $>70 \%$ for RCA and LCX in comparison with the LAD. A patientbased analysis is shown in Figure 2B, revealing that if a patient had a coronary lesion $>70 \%, 76 \%(13 / 17)$ of them showed myocardial ischemia. Figure $2 \mathrm{C}$ shows the correlation for increasing extent of $\mathrm{CAD}$ and ischemia: $<50 \%, 9 \%$ (2/23); 1 vessel with $\geq 50 \%$ stenosis, $18 \%$ (5/ 28); 2 vessels with $\geq 50 \%$ stenosis, $46 \%(6 / 13)$ and 3 vessels with $\geq 50 \%$ stenosis, $78 \%$ (7/9).

\section{DISCUSSION}

The main findings of the present analysis are as follows: increasing stenosis percentage measured with quantitative CTA relates to an increasing percentage of myocardial ischemia measured with adenosine stress myocardial CTP. Second, the current quantitative CTA analysis demonstrated that hemodynamically significant lesions comprised a higher plaque and dense calcium volume, mean plaque burden, larger maximal plaque thickness, and longer lesion length, but only stenosis severity and lesion length were independent determinants.

Table 3. Quantitative CTA parameters for ischemic and non-ischemic lesions

\begin{tabular}{lccr}
\hline Quantitative CTA parameters $^{\mathbf{a}}$ & Ischemia $(\mathbf{n}=\mathbf{3 1})$ & No ischemia $(\mathbf{n}=\mathbf{1 1 5})$ & $\boldsymbol{P}$ value \\
\hline Lumen area stenosis (\%) & $69.0 \pm 16.8$ & $49.6 \pm 17.2$ & $<.001$ \\
Lumen diameter stenosis (\%) & $46.7 \pm 16.8$ & $30.2 \pm 13.1$ & $<.001$ \\
Mean plaque burden (\%) & $59.6 \pm 8.7$ & $52.3 \pm 9.9$ & $<.001$ \\
Plaque volume $\left(\mathrm{mm}^{3}\right)$ & $68.7(40.2-126.0)$ & $44.7(24.1-80.0)$ & .021 \\
Lesion length (mm) & $12.3(4.9-14.9)$ & $6.5(4.0-10.4)$ & .033 \\
Maximal plaque thickness (mm) & $2.3(1.8-2.7)$ & $1.9(1.5-2.4)$ & .021 \\
Fibrous volume $\left(\mathrm{mm}^{3}\right)$ & $24.7(14.2-43.4)$ & $16.9(9.6-32.9)$ & .068 \\
Fibro-fatty volume $\left(\mathrm{mm}^{3}\right)$ & $3.9(1.7-8.3)$ & $3.6(1.5-9.9)$ & .928 \\
Necrotic core volume $\left(\mathrm{mm}^{3}\right)$ & $0.6(0.1-2.1)$ & $0.9(0.1-1.9)$ & .661 \\
Dense calcium volume $\left(\mathrm{mm}^{3}\right)$ & $25.8(7.6-39.4)$ & $7.8(0.0-20.2)$ & .005 \\
Remodeling index & $1.0(0.9-1.1)$ & $1.0(0.9-1.1)$ & .820
\end{tabular}

CTA, Computed tomography angiography.

${ }^{a}$ Results from the most severe lesion per coronary artery. 


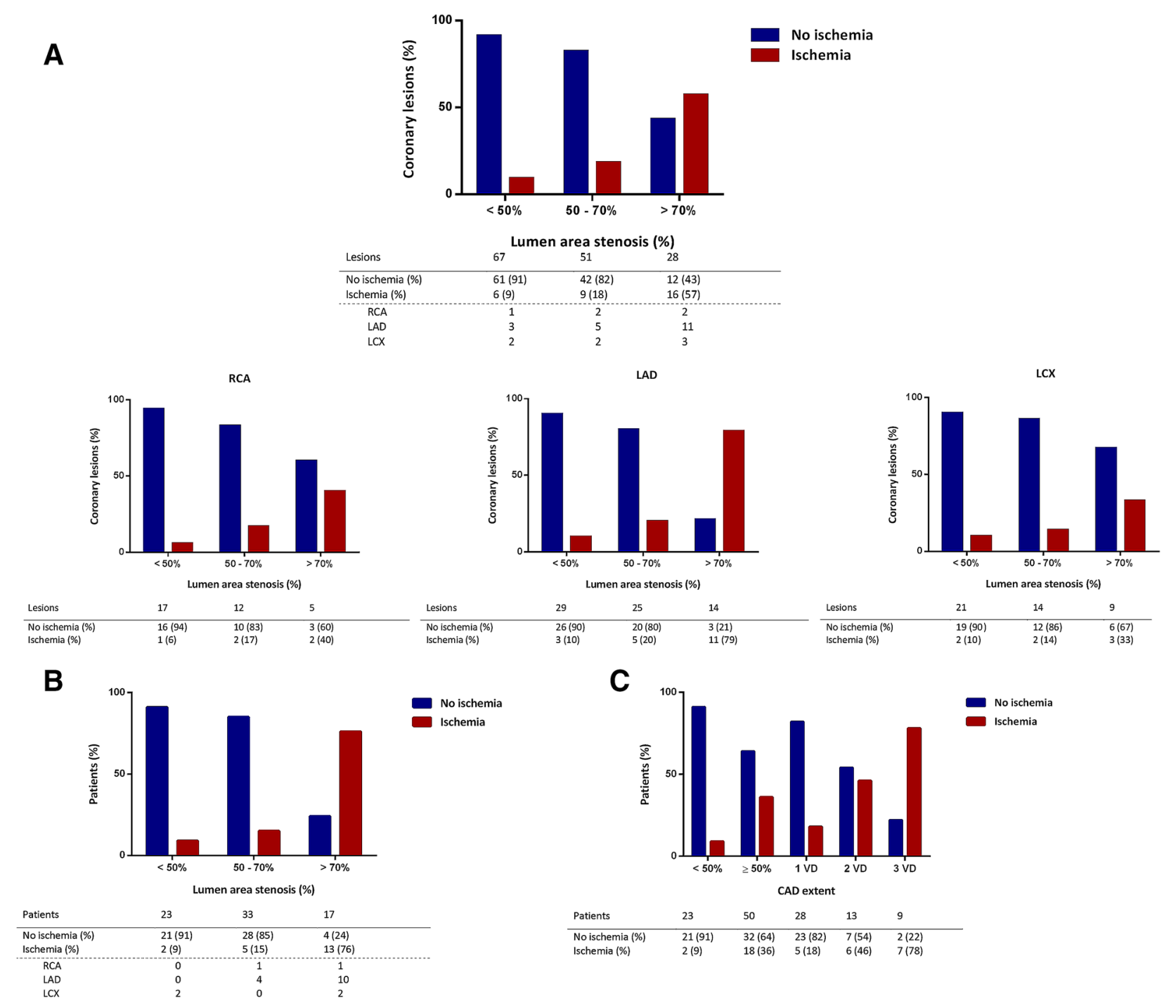

Figure 2. (A) Vessel-based analysis relating stenosis percentage to myocardial ischemia. (B) Patient-based analysis relating stenosis percentage to myocardial ischemia. (C) Extent of CAD related to myocardial ischemia. $C A D$, Coronary artery disease; $V D$, vessel with $\geq 50 \%$ stenosis.

\section{Stenosis Severity Vs Myocardial Ischemia}

The relation between stenosis severity and myocardial ischemia has been investigated previously. ${ }^{11,14,15}$ Uren et al related the percentage stenosis of a coronary lesion to downstream myocardial blood flow measured with positron emission tomography (PET) and demonstrated that basal myocardial blood flow during rest remained constant regardless of stenosis severity. ${ }^{11}$ Moreover, only in lesions with $\geq 40 \%$ stenosis, myocardial flow progressively decreased during hyperaemia, indicating that lesions below $40 \%$ stenosis have no hemodynamic consequences. More studies confirmed this inverse relation between hyperaemic blood flow and stenosis severity and quantified the exact percentage of stenosis to the presence of myocardial ischemia. ${ }^{16,17} \mathrm{Di}$ Carli et al demonstrated a significantly lower flow reserve (as measured with PET) in lesions with stenosis between $70 \%$ and $90 \%$ as compared to $50 \%$ and $70 \%{ }^{16}$ While stenosis severity had only a moderate impact on downstream hyperaemic myocardial flow, no difference in flow reserve was observed in lesions of $50 \%-70 \%$ compared with lesions below $50 \%$.

More recently, studies using invasive FFR for assessment of hemodynamic consequences of a stenosis, 
confirmed that moderate lesions do not often result in myocardial ischemia. Tonino et al demonstrated with quantitative coronary angiography that lesions with a stenosis degree of 50\%-70\%, 71\%-90\%, and 91\%-99\%, respectively, $35 \%, 80 \%$, and $96 \%$ showed a FFR $\leq 0.80 .{ }^{18}$ In accordance with ICA, coronary CTA is of limited value in predicting the hemodynamic significance of a stenotic lesion. Schuijf et al reported that in 114 patients who underwent coronary CTA and single-photon emission-computed tomography (SPECT) myocardial perfusion imaging, only $50 \%$ of obstructive coronary lesions were ischemia-related. ${ }^{3}$ Similar findings were reported by Sato et al who established that for 105 coronary lesions with $60 \%-70 \%, 70 \%-80 \%$, and $\geq 80 \%$ stenosis, the prevalence of ischemia was, respectively, 33\%, 54\%, and $86 \% .{ }^{19}$ Thus, whether assessed by ICA or coronary CTA, an intermediate to severe stenosis degree of a coronary lesion provides insufficient information for clinical decision making and requires further ischemia testing. Therefore, integration of coronary CTA with the assessment of myocardial ischemia in one session would be ideal, which has become feasible with adenosine stress myocardial CTP. George et al demonstrated the feasibility of adenosine stress myocardial CTP to detect myocardial ischemia. ${ }^{20}$ In a sub-study of the CORE320 trial in which 381 patients underwent adenosine myocardial stress CTP and SPECT myocardial perfusion imaging, CTP yielded a higher sensitivity (88\% [CI 83-92] to 62 [CI 56-69]), but a lower specificity (55\% [CI 46-63] to 67 [CI 59-75] to predict obstructive CAD ( $\geq 50 \%$ stenosis) measured with ICA. ${ }^{21}$

Hence, with the integrated assessment of coronary CTA and adenosine stress myocardial CTP, reliable information concerning stenosis severity and corresponding myocardial perfusion (and ischemia) can be obtained in one session. In the current study, we related quantitatively assessed coronary stenosis severity to myocardial ischemia assessed by adenosine stress myocardial CTP. In accordance to previously mentioned results, we demonstrated a moderate association between intermediate and severely stenotic lesions and myocardial ischemia, especially for the RCA and LCX. The detection of myocardial ischemia by stress myocardial CTP related to coronary anatomy has not been investigated previously. These findings may encourage to perform stress myocardial CTP after coronary CTA throughout a wide range of stenosis degree on coronary CTA.

\section{Adenosine Stress Myocardial CTP Vs Coronary CTA Plaque Composition}

An explanation of these findings could be that obstructive CAD is just one manifestation of atherosclerosis. Other mechanisms as coronary vasospasm, inflammation, microvascular dysfunction, endothelial dysfunction, and thrombosis relate to myocardial ischemia as well. ${ }^{22}$ Besides stenosis severity, plaque composition and morphology may also contribute to the development of ischemia. Indeed, recent studies demonstrated that certain plaque characteristics were independently associated with the presence of ischemia. ${ }^{6,23,24}$

First, Park et al reported the incremental value of spotty calcifications, low attenuation plaque, positive remodeling, and percent aggregate plaque volume (\% APV) to predict ischemia. ${ }^{6} \% \mathrm{APV}$ represents the total plaque volume as function of total vessel volume from the ostium till the distal part of the lesion, indicating the patient's total atherosclerotic burden. Nakazato et al confirmed the independent and incremental value of $\%$ APV for ischemia (assessed by FFR). ${ }^{23}$

Second, Naya et al reported that ischemia measured with PET correlated with the modified Duke CAD index (which indicates the total atherosclerotic burden in a patient). ${ }^{25}$ The relation between diffuse coronary atherosclerosis and ischemia was emphasized earlier by De Bruyne et al. ${ }^{26}$

Our findings show that the plaque characteristics representing total atherosclerotic burden were higher in ischemic lesions, but only stenosis severity and lesion length were independent determinants for ischemia. Although promising, the exact cut-off values for new plaque parameters should be investigated more extensively in future studies.

\section{LIMITATIONS}

As adenosine stress myocardial CT perfusion has only recently been implemented, the small sample size of this single center analysis is a limitation. With this first-pass contrast enhancement technique, acquisition timing is crucial to be able to detect attenuation differences between ischemic and normal myocardium. Furthermore, most patients only underwent adenosine stress myocardial CTP if obstructive CAD was suspected based on visual analysis, which could have introduced selection bias by creating a population with high CAD burden. Interpretation of adenosine stress myocardial CTP images is challenging, mainly due to the presence of motion and beam-hardening artifacts which can mimic and mask hypo-perfusion, potentially reducing diagnostic accuracy. Although studies of insufficient quality were excluded, image artifacts could have biased our results.

\section{NEW KNOWLEDGE GAINED}

This study explored the relationship between coronary CTA-derived plaque characteristics and ischemia 
by stress myocardial CTP. Quantitatively measured stenosis severity was moderately correlated with ischemia. Stenosis severity and lesion length were independent correlates, adjusted for mean plaque burden, plaque volume, maximal plaque thickness, and dense calcium volume.

\section{CONCLUSION}

Increasing stenosis percentage by quantitative CTA is positively correlated to myocardial ischemia measured with adenosine stress myocardial CTP. However, stenosis percentage remains a moderate determinant for ischemia.

Coronary plaque volume, mean plaque burden, lesion length, maximal plaque thickness, and dense calcium volume were all significantly associated with myocardial ischemia, but only lesion length and stenosis severity were independent determinants.

\section{Acknowledgments}

Alexander R. van Rosendael is supported by a research grant from the Interuniversity Cardiology Institute of the Netherlands (ICIN, Utrecht, The Netherlands). The Department of Cardiology received research grants from Biotronik, Medtronic, Boston Scientific Corporation, St. Jude Medical, Lantheus Medical Imaging and GE Healthcare.

\section{Disclosure}

The authors have indicated that they have no financial conflict of interest.

\section{Open Access}

This article is distributed under the terms of the Creative Commons Attribution 4.0 International License (http://creativecommons.org/licenses/by/4.0/), which permits unrestricted use, distribution, and reproduction in any medium, provided you give appropriate credit to the original author(s) and the source, provide a link to the Creative Commons license, and indicate if changes were made.

\section{References}

1. Miller JM, Rochitte CE, Dewey M, Arbab-Zadeh A, Niinuma H, Gottlieb I, et al. Diagnostic performance of coronary angiography by 64-row CT. N Engl J Med 2008;359:2324-36.

2. Hoffmann MH, Shi H, Schmitz BL, Schmid FT, Lieberknecht M, Schulze R, et al. Noninvasive coronary angiography with multislice computed tomography. JAMA 2005;293:2471-8.

3. Schuijf JD, Wijns W, Jukema JW, Atsma DE, de RA, Lamb HJ, et al. Relationship between noninvasive coronary angiography with multi-slice computed tomography and myocardial perfusion imaging. J Am Coll Cardiol 2006;48:2508-14.
4. Hachamovitch R, Berman DS, Shaw LJ, Kiat H, Cohen I, Cabico $\mathrm{JA}$, et al. Incremental prognostic value of myocardial perfusion single photon emission computed tomography for the prediction of cardiac death: Differential stratification for risk of cardiac death and myocardial infarction. Circulation 1998;97:535-43.

5. de Graaf MA, El-Naggar HM, Boogers MJ, Veltman CE, Broersen A, Kitslaar PH, et al. Automated quantitative coronary computed tomography correlates of myocardial ischaemia on gated myocardial perfusion SPECT. Eur J Nucl Med Mol Imaging 2013;40:1171-80.

6. Park HB, Heo R, Hartaigh O, Cho I, Gransar H, Nakazato R, et al. Atherosclerotic plaque characteristics by $\mathrm{CT}$ angiography identify coronary lesions that cause ischemia: A direct comparison to fractional flow reserve. JACC Cardiovasc Imaging 2015;8:1-10.

7. Boogers MJ, Broersen A, van Velzen JE, de Graaf FR, El-Naggar $\mathrm{HM}$, Kitslaar PH, et al. Automated quantification of coronary plaque with computed tomography: Comparison with intravascular ultrasound using a dedicated registration algorithm for fusionbased quantification. Eur Heart J 2012;33:1007-16.

8. Rochitte CE, George RT, Chen MY, Arbab-Zadeh A, Dewey M, Miller JM, et al. Computed tomography angiography and perfusion to assess coronary artery stenosis causing perfusion defects by single photon emission computed tomography: The CORE320 study. Eur Heart J 2014;35:1120-30.

9. Hausleiter J, Meyer T, Hermann F, Hadamitzky M, Krebs M, Gerber TC, et al. Estimated radiation dose associated with cardiac CT angiography. JAMA 2009;301:500-7.

10. de Graaf MA, Broersen A, Kitslaar PH, Roos CJ, Dijkstra J, Lelieveldt BP, et al. Automatic quantification and characterization of coronary atherosclerosis with computed tomography coronary angiography: Cross-correlation with intravascular ultrasound virtual histology. Int J Cardiovasc Imaging 2013;29:1177-90.

11. Uren NG, Melin JA, De BB, Wijns W, Baudhuin T, Camici PG. Relation between myocardial blood flow and the severity of coronary-artery stenosis. N Engl J Med 1994;330:1782-8.

12. Cerqueira MD, Weissman NJ, Dilsizian V, Jacobs AK, Kaul S, Laskey WK, et al. Standardized myocardial segmentation and nomenclature for tomographic imaging of the heart. A statement for healthcare professionals from the Cardiac Imaging Committee of the Council on Clinical Cardiology of the American Heart Association. J Nucl Cardiol 2002;9:240-5.

13. Mehra VC, Valdiviezo C, Arbab-Zadeh A, Ko BS, Seneviratne SK, Cerci R, et al. A stepwise approach to the visual interpretation of CT-based myocardial perfusion. J Cardiovasc Comput Tomogr 2011;5:357-69.

14. Kirkeeide RL, Gould KL, Parsel L. Assessment of coronary stenoses by myocardial perfusion imaging during pharmacologic coronary vasodilation. VII. Validation of coronary flow reserve as a single integrated functional measure of stenosis severity reflecting all its geometric dimensions. J Am Coll Cardiol 1986;7:103-13.

15. de Bruyne B, Baudhuin T, Melin JA, Pijls NH, Sys SU, Bol A, et al. Coronary flow reserve calculated from pressure measurements in humans. Validation with positron emission tomography. Circulation 1994;89:1013-22.

16. di Carli M, Czernin J, Hoh CK, Gerbaudo VH, Brunken RC, Huang SC, et al. Relation among stenosis severity, myocardial blood flow, and flow reserve in patients with coronary artery disease. Circulation 1995;91:1944-51.

17. Demer LL, Gould KL, Goldstein RA, Kirkeeide RL, Mullani NA, Smalling RW, et al. Assessment of coronary artery disease severity by positron emission tomography. Comparison with quantitative arteriography in 193 patients. Circulation 1989; 79:825-35. 
18. Tonino PA, Fearon WF, De BB, Oldroyd KG, Leesar MA, Ver Lee PN, et al. Angiographic versus functional severity of coronary artery stenoses in the FAME study fractional flow reserve versus angiography in multivessel evaluation. J Am Coll Cardiol 2010;55:2816-21.

19. Sato A, Hiroe M, Tamura M, Ohigashi H, Nozato T, Hikita H, et al. Quantitative measures of coronary stenosis severity by 64 slice CT angiography and relation to physiologic significance of perfusion in nonobese patients: Comparison with stress myocardial perfusion imaging. J Nucl Med 2008;49:564-72.

20. George RT, Arbab-Zadeh A, Miller JM, Kitagawa K, Chang HJ, Bluemke DA, et al. Adenosine stress 64- and 256-row detector computed tomography angiography and perfusion imaging: A pilot study evaluating the transmural extent of perfusion abnormalities to predict atherosclerosis causing myocardial ischemia. Circ Cardiovasc Imaging 2009;2:174-82.

21. George RT, Mehra VC, Chen MY, Kitagawa K, Arbab-Zadeh A, Miller JM, et al. Myocardial CT perfusion imaging and SPECT for the diagnosis of coronary artery disease: A head-to-head comparison from the CORE320 multicenter diagnostic performance study. Radiology 2014;272:407-16.
22. Marzilli M, Merz CN, Boden WE, Bonow RO, Capozza PG, Chilian WM, et al. Obstructive coronary atherosclerosis and ischemic heart disease: An elusive link! J Am Coll Cardiol 2012;60:951-6.

23. Nakazato R, Shalev A, Doh JH, Koo BK, Gransar H, Gomez MJ, et al. Aggregate plaque volume by coronary computed tomography angiography is superior and incremental to luminal narrowing for diagnosis of ischemic lesions of intermediate stenosis severity. J Am Coll Cardiol 2013;62:460-7.

24. Diaz-Zamudio M, Dey D, Schuhbaeck A, Nakazato R, Gransar H, Slomka PJ, et al. Automated quantitative plaque burden from coronary CT angiography noninvasively predicts hemodynamic significance by using fractional flow reserve in intermediate coronary lesions. Radiology 2015;276:408-15.

25. Naya M, Murthy VL, Blankstein R, Sitek A, Hainer J, Foster C, et al. Quantitative relationship between the extent and morphology of coronary atherosclerotic plaque and downstream myocardial perfusion. J Am Coll Cardiol 2011;58:1807-16.

26. de Bruyne B, Hersbach F, Pijls NH, Bartunek J, Bech JW, Heyndrickx GR, et al. Abnormal epicardial coronary resistance in patients with diffuse atherosclerosis but "Normal" coronary angiography. Circulation 2001;104:2401-6. 